Société d'histoire de la révolution de 1848 et des

révolutions du XIXe siècle

49 | 2014

1814-1815. Expériences de la discontinuité

\title{
Le département du Mont-Blanc en 1814-1815. Nationalité et Restaurations en situation de frontière
}

The départment of Mont-Blanc in 1814-1815. Nationality and Restorations in a situation of borders

Das département Mont-Blanc 1814 und 1815. Nationalität und Restauration im Grenzgebiet

Sylvain Milbach

\section{CpenEdition}

Journals

Édition électronique

URL : http://journals.openedition.org/rh19/4746

DOI : $10.4000 /$ rh 19.4746

ISSN : $1777-5329$

Éditeur

La Société de 1848

Édition imprimée

Date de publication : 1 décembre 2014

Pagination : 65-78

ISSN : 1265-1354

Référence électronique

Sylvain Milbach, «Le département du Mont-Blanc en 1814-1815. Nationalité et Restaurations en situation de frontière », Revue d'histoire du XIXe siècle [En ligne], 49 | 2014, mis en ligne le 01 décembre 2014, consulté le 21 avril 2019. URL : http://journals.openedition.org/rh19/4746 ; DOI : 10.4000/ rh19.4746 


\section{SYLVAIN MILBACH}

\section{Le département du Mont-Blanc en 1814-1815. Nationalité et Restaurations en situation de frontière}

"Venez, Sire, venez reprendre sur les fondateurs du Trône de vos ayeux [sic] l'exercice des droits qui ont fait si longtemps leur félicité : ce sont des enfants soumis qui appellent un père tendre; des temps malheureux les ont séparés de vous, mais ils n'ont pu effacer l'affection dont ils étaient pénétrés pour votre auguste famille; vous la retrouverez, Sire, toujours la même et plus vive encore» ${ }^{1}$.

«Braves habitants des Alpes, race illustre des Allobroges : [...] vous vous retracerez cette époque désastreuse où la complaisante faiblesse d'un monarque vous faisait entrevoir dans l'avenir un joug honteux d'un despote oublié, étranger à notre siècle, à nos mœurs, à notre langue, et qui fait régner avec lui les préjugés et l'ignorance. Vos craintes vous reportaient aux temps de l'inquisition, du servage et des ceps; vous entendiez le sifflement des verges nobiliaires, et ces voix superbes qui vous criaient: "Peuple! Les rois sont vos divinités, vous n'êtes ici-bas que pour leurs menus plaisirs". Alors vous leviez vos fronts pâlis d'indignation $»^{2}$.

Après vingt-deux ans de régime français, l'ex-duché de Savoie, annexé dès 1792, réunissait un certain nombre de conditions pour entrer dans une phase de violents antagonismes où des mémoires concurrentes pouvaient être mises au service d'intérêts contradictoires. D'ailleurs, dans les rapports des préfets, deux partis sont nettement identifiés dès $1814^{3}$ : d'un côté, une partie de la classe aristocratique qui penche pour le retour du duché sous le sceptre du roi de Sardaigne, lequel s'oriente dès 1814 vers une restauration absolue ${ }^{4}$; de l'autre, la classe bourgeoise attachée à ses positions nées de la Révolution.

1. Adresse au roi de Sardaigne, Archives départementales de Savoie (Arch. dép. Savoie), L 1429, procès-verbaux de la commission centrale du Mont-Blanc, 29 avril 1814.

2. Le nouveau cri des Alpes, Chambéry, Gorrin, 1815, p. 5. L'auteur de cet appel martial, daté de mai 1815, est Constant Durhone, officier de l'état-major du général Dessaix. Il reprend le titre d'un opuscule célèbre paru dans la Savoie pré-révolutionnaire.

3. Archives nationales (Arch. nat.), essentiellement: F ${ }^{1 \text { cIII }}$ Mont-Blanc 3; F ${ }^{\text {cIII }}$ Léman 2; $\mathrm{F}^{\text {1bII }}$ Mont-Blanc 4; F ${ }^{2 \mathrm{I}} 862 ; \mathrm{F}^{7} 7022$.

4. Sur la restauration sarde: Ombre e luci della Restaurazione. Trasformazioni e continuità 
Pourtant, tout en gardant la trace de ces divergences au sein des élites, les rapports préfectoraux comme les archives qui consignent l'activité des autorités locales 5 ne laissent voir que fort peu de troubles, ne trahissent aucun symptôme sérieux de guerre civile et ne cessent de louer le bon esprit du "peuple de Savoie». Sans doute, peut-on relever dans les archives comme dans l'érudition locale quelques épisodes qui manifestent des tensions, des résistances fiscales surtout, mais jamais l'ordre ne parait menacé. En revanche, l'inertie semble forte et le préfet rend compte de la grande difficulté d'administrer un département où les élites rechignent à prendre des charges officielles ${ }^{6}$, pour ne rien dire de la difficulté à lever des hommes en 1814 comme en 1815 .

Entre janvier 1814, quand les troupes autrichiennes pénètrent la Savoie, et décembre 1815, quand l'intégralité de l'ancien duché retourne au roi de Sardaigne, les Savoyards ne cessent de connaître la précarité. Celle des opérations militaires au début de l'année 1814, puis lors des Cent-Jours; celle de l'occupation à la suite de ces opérations; celle enfin qui résulte de l'incertitude sur l'avenir du duché, entre France, Suisse et Piémont ${ }^{7}$. Les populations n'eurent pas à souffrir excessivement des opérations militaires en tant que telles et les affrontements directs furent d'ailleurs rares ${ }^{8}$. En revanche, l'occupation, comme en témoignent les archives préfectorales et celles des commissions nommées par les autorités autrichiennes ${ }^{9}$, pesa lourd avec son cortège habituel de misères : réquisitions en nature, épizooties, impôts exceptionnels, etc. ${ }^{10}$ La première Restauration et les Cent-Jours surtout ne furent pas vécus comme des temps de pause et on ne peut pas dire que les changements de régimes aient signifié des ruptures en termes de pression fiscale notamment. Ces presque deux années, de janvier 1814 à la fin de l'été 1815, apparaissent donc comme des temps d'exception.

Ce climat d'incertitude ne put qu'être vivifié par le premier règlement territorial qui fit suite au premier traité de Paris : l'ancien territoire savoyard

istituzionali nei terrori del Regno di Sardegna, Rome, Ministero per i beni culturali e ambientali, Ufficio centrale per i beni archivistici, 1997.

5. Arch. dép. Savoie, parmi des sources assez abondantes: L 123, lettres envoyées par l'administration départementale aux ministres; L 1428, ordres et proclamations des chefs de l'armée autrichienne; L 1429-1430, Commission centrale du département du Mont-Blanc, arrêtés et pièces diverses; L 1437-42, commissions subsidiaires d'Annecy et de Chambéry, arrêtés et correspondance; L 1462, ordres, proclamations et lettres des généraux et officiers de l'armée d'invasion; L 1463-1464, Commission départementale du Mont-Blanc, procès-verbaux; L 1472, lettres et circulaires envoyées par le préfet, instructions ministérielles, états et affaires diverses.

6. Arch. nat., FibII Mont-Blanc 4, extrait d'une lettre du préfet Finot, 13 mai 1814.

7. Sur cette période : André Palluel-Guillard, Une fusion manquée. Genève et la Savoie dans le grand empire napoléonien, 1799-1815, thèse d'Etat sous la direction de Jean Tulard, Université de Savoie, 1991 , tome II.

8. Les opérations militaires en Savoie sont bien connues, on se reportera au récit et à la bibliographie de la thèse d'André Palluel-Guillard.

9. Les Autrichiens déléguèrent à une Commission centrale, dite du Mont-Blanc, épaulée par des commissions subsidiaires au niveau des arrondissements, les levées nécessaires à l'entretien des troupes d'occupation.

10. Voir le compte très lourd et méticuleux du préfet Finot : Rapport adressé par M. le préfet du Mont-Blanc..., relativement aux mesures administratives auxquelles a donné lieu l'occupation du territoire

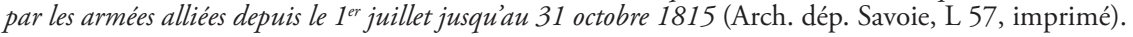


était divisé suivant une frontière nord-sud parfaitement arbitraire et ne correspondant à aucun héritage historique, aucune logique géographique; à l'est de cette ligne, s'installent les autorités sardes, tandis qu'à l'ouest était créé un nouveau département du Mont-Blanc (180000 habitants, dont 30000 de l'ex-département du Léman, sur environ 450000 Savoyards). Le préfet prend la tête de ce nouveau département en juin 1814. Si les Savoyards étaient accoutumés de longue date aux occupations, voire aux changements de régimes ${ }^{11}$, ce partage créait une situation inédite. Nombreux étaient en effet les intérêts divisés par cette frontière qui ne respectait ni les familles, ni les propriétés, ni les logiques territoriales et économiques. C'était vrai pour l'élite : aristocratie et bourgeoisie s'accordaient pour se plaindre de la séparation entre leurs lieux de résidence urbaine, qui se trouvaient dans un royaume, et leurs villégiatures et propriétés foncières, qui se trouvaient dans un autre. Les grands propriétaires de vignobles de la Combe de Savoie s'inquiétaient de devoir payer les droits de douanes pour rapatrier leur production à Chambéry. Mais cette situation affectait aussi une grande partie de la population. Ainsi, toutes les vallées étaient coupées de l'avant-pays, donc de leurs débouchés naturels pour leur production; singulièrement au nord de la Savoie, ces vallées étaient coupées de Genève. Cette situation était ingérable et le préfet du Mont-Blanc était le premier à s'en plaindre.

Cette frontière et son curieux mais très provisoire règlement devaient contribuer à exacerber de manière originale et précoce la "question nationale». Il ne semble pas qu'elle se soit posée avec autant d'acuité en d'autres zones frontalières : à la différence des départements rhénans ${ }^{12}$, les armées d'occupation ne se sont livrées à aucune espèce de propagande visant à enrôler les populations; les Autrichiens étaient peu intéressés en Savoie et se sont limités à conseiller le calme et à faire lever «l'impôt». Il n'y a pas eu dans les départements rhénans de mouvement pro-français et, dans les départements belges par exemple, la séparation se fit sans réels débats ${ }^{13}$. Il en va autrement en Savoie où les autorités occupantes ont adopté une position encore plus hésitante qu'ailleurs. Dans sa première proclamation, lors de son entrée en Savoie, le général Bubna laissait clairement entendre qu'elle serait rendue à son souverain légitime ${ }^{14}$; mais, arrivé à Chambéry, il éloigne les représentants de la noblesse pro-sarde au profit de notables qui ont administré le département sous l'Empire. Bubna écartait ainsi le général de Sonnaz qui

11. Frédéric Meyer, «Occupations ou annexions? La Savoie soumise. 1536-1749», in Sylvain Milbach [dir.], La Savoie, terre ouverte. Occupations, annexions, révolutions, Chambéry, SSHA, 2010, p. 13-35.

12. Roger Dufraisse, «La fin des départements de la rive gauche du Rhin », in Yves-Marie Bercé [dir.], La fin de l'Europe napoléonienne. 1814: la vacance du pouvoir, Paris, Henri Veyrier, 1990, p. 11-61.

13. Paul Janssens, «Le changement de régime dans les départements belges», ibidem, p. 79-107.

14. Proclamation du 2 avril 1814 (Arch. dép. Savoie, L 1428) : «Le retour de vos princes est prochain». 
avait levé des troupes ${ }^{15}$, appelé la Savoie au soulèvement, pris langue dès janvier 1814 avec le quartier général autrichien et accompagné les troupes jusqu’à Chambéry. D'Oncieu ${ }^{16}$, maire de Chambéry, se voyait de son côté confirmé dans son mandat et il lui était même confié l'administration de l'ensemble de la zone occupée; bel exemple de continuité administrative ${ }^{17}$, qu'on retrouve à la préfecture, avec le préfet Finot qui sera rétabli sous les deux Restaurations, et pour partie dans les commissions nommées par l'occupant, et qui contribue peut-être à expliquer l'absence de troubles majeurs malgré les changements de régimes.

Puisque les autorités "naturelles» du duché, c'est-à-dire les grandes familles, étaient ainsi tenues à distance relative de l'administration du territoire, il leur revenait de prendre l'offensive en faveur de la monarchie sarde par d'autres moyens. C'est sur ce point que la séquence 1814-1815 en Savoie présente une originalité forte : si sa noblesse n'avait rien oublié, on ne peut pas dire qu'elle n'avait rien appris. Sur ce point, nous sommes convaincus que cette séquence ne peut se résumer, malgré toutes les apparences, à un pur et simple retour à l'ordre ancien des choses, mais participe bien, par la paradoxale continuité du discours et des pratiques surtout, du travail long et sourd de la Révolution à l'heure des restaurations. Le règlement de la question se déroule simultanément à plusieurs échelles : les tractations internationales, les intérêts de l'élite locale, dont une partie est en contact avec le monde diplomatique, et ceux des populations. S'il ne relève pas de nos perspectives de retracer les tractations internationales où se jouèrent d'abord les destinées du duché ${ }^{18}$, il convient en revanche de sonder comment leur lenteur, qui se traduit en Savoie par une difficile et hésitante normalisation, permit et encouragea à user du levier de l'opinion. Pour bien comprendre les mécanismes que fait jouer la situation de frontière, il convient en effet de mesurer combien la mobilisation des élites s'articule avec un contexte d'incertitude qui affecte le quotidien de la population. Cette incertitude contribue à créer un climat d'attente et une effervescence de l'attention en mesure de justifier la diffusion d'un argumentaire de type nationalitaire. Le ralliement au régime de restauration sarde se justifie en Savoie par la convocation du sentiment national et de l'opinion.

15. Janus Gerbaix de Sonnaz (1736-1814), d'une grande famille de la noblesse savoyarde, de tradition militaire.

16. Jean-Baptiste d'Oncieu (1765-1847), officier piémontais démobilisé en 1798; maire de Chambéry en 1813, il connait ensuite une belle carrière administrative sous la restauration sarde, bien qu'il fût un serviteur fidèle du régime français.

17. Cette question a été récemment revisitée : Marie-Cécile Thoral, «L'administration locale en temps de crise : le cas de l'Isère en 1814-1815", Annales historiques de la Révolution française, n 339 , 2005, p. 117-135.

18. Là encore, voir André Palluel-Guillard, Une fusion manquée..., op. cit. 
«ON a débité I OOO ET I OOO NOUVElles ET IL S’EST FAIt UnE INFINITÉ DE COMPTES SUR LE SORT DE LA SAVOIE » ${ }^{19}$

Si les sources administratives laissent assez bien saisir comment vécurent les Savoyards durant ces deux années, il est infiniment plus difficile de se faire une idée de ce qu'ils pensèrent. Les rapports des préfets ou les rapports des envoyés extraordinaires sont à la fois peu précis et, on l'a dit, peu inquiets en ce qui concerne l'attitude des populations. À l'heure de la «naissance» de l'esprit public ${ }^{20}$, il paraît clair seulement qu'il convient d'alléger les charges d'une province exsangue et de faciliter ainsi son ralliement. Il est en revanche possible, à partir du cas chambérien, de mesurer le désordre jeté dans le quotidien, même si on reste dans le milieu des notables urbains.

Dans cette optique, on dispose d'un témoignage inestimable, le journal laissé par l'avocat Charles Mansord, tenu de janvier 1814 à décembre $1815^{21}$. Ce qui se dégage avant tout de ses notes, c'est que la guerre et l'occupation se traduisent par un desserrement administratif qui contraste avec la période impériale. On voit bien aussi comment s'enchevêtrent dans les considérations de Mansord les différentes échelles de la réalité, qui sont aussi des temporalités différentes : attention évidemment aux opérations militaires sur le territoire savoyard, mais aussi en France; consignation de toute information sur les tractations diplomatiques; attention à l'attitude du microcosme chambérien. Le journal bruit ainsi de toutes sortes d'informations, éphémères, vraies et plus souvent fausses. On sent à lire l'avocat que presque sans discontinuité de janvier 1814 à décembre 1815, l'avenir de la Savoie n'est jamais assuré, même durant les périodes de rétablissement d'une administration officielle. Ce n'est pourtant pas l'angoisse qui sourd du journal de Mansord, mais une sorte de frénésie, une attente perpétuelle de nouvelles qui autoriseraient à décrypter l'avenir. La rumeur traduit ce desserrement et cet état d'esprit assez général.

Quelles sont ses sources d'information? La presse officielle apparaît très peu dans le journal de Mansord, alors que le Journal du département du MontBlanc, alternativement journal officiel de la préfecture et des autorités d'occupation, paraît assez régulièrement durant cette période et publie sans états d'âme les avis officiels des autorités qui se succèdent. Cet asservissement peut expliquer le peu d'attrait qu'il suscite, mais c'est surtout que cet hebdoma-

19. Arch. dép. Savoie, Charles Mansord, «Journal concernant les événements des 1814-1816 en Savoie», manuscrit, J 1.

20. Pierre Karila-Cohen, L'État des esprits. L'invention de l'enquête politique en France (1814-1848), Rennes, Presses universitaires de Rennes, 2008.

21. Charles Mansord (1756-1832) laisse un manuscrit inédit (note 19, ci-dessus), en fait une copie réalisée par un érudit local, Henry Carle. Ce journal est tenu presque jour après jour du 20 janvier 1814 au 18 décembre 1815, avec toutefois un large manque entre juillet 1814 et mars 1815 (coupe du copiste ou suspension du journal durant la première Restauration?). Avocat et juriste réputé, Charles Mansord avait été maire de Chambéry en 1792, magistrat sous l'Empire, où il se démit de ses charges administratives. Il fut toutefois sollicité en 1814-1815 pour faire partie des commissions qui administrèrent le département occupé. 
daire n'épouse pas la temporalité des événements; sous cet angle, la rumeur comble mieux l'impatience. Le périodique est à peine plus utile à l'heure des occupations ou de l'installation des nouvelles autorités où la voie d'affichage public devient le moyen d'information en temps réel et, notons-le, les interprétations possibles de cet affichage, de nouvelles sources de rumeurs : il ne vient jamais à bout de l'attente ${ }^{22}$. Les affichages sauvages, manuscrits ou imprimés, parfois encadrés des couleurs nationales adéquates, retiennent toute l'attention, plus que l'affichage officiel qui dans ses platitudes administratives parait toujours décevant. Â cette source Mansord préfère le courrier autour duquel on se presse : poste qui apporte affiches ou Moniteur; plus encore, courrier qui renseigne par la voie de son agent («Le 12 [avril 1814] au matin le courrier de Lyon est arrivé avec la cocarde blanche») ou des voyageurs qui colportent les nouvelles les plus fraîches, généralement venues de Lyon; lettres privées enfin, qui racontent aussi tout et son contraire, mais dont le crédit est fort tant l'information officielle ne paraît pas au diapason de la dilatation des possibles qui naissent de l'incertitude. Le tout «fait tenir tous les raisonnements et toutes les conjectures». Lorsque le courrier est suspendu, Mansord est obligé de faire appel à des sources moins «sûres». Parmi les signes qui lui paraissent les plus probants, la valse des couleurs figure parmi les plus significatifs et elle interroge même sur la conduite à tenir : «Le 11 [juin 1814] grands débats pour savoir si demain à la procession [de la Fête-Dieu] l'on portera la cocarde bleue ou la cocarde blanche». Les insignes partisans, jamais arborés simultanément semble-t-il car les autorités y veillent sévèrement, sont considérés comme de bons thermomètres de la situation, dont ils indiquent surtout l'instabilité et le manque concret d'information : les cocarde tricolores des Cent-Jours, les blanches de la Restauration française et les bleues de la Maison de Savoie; les croix de Savoie que les femmes portent en sautoir et les hommes au revers; les drapeaux bien sûr, signes officiels des changements de régimes, mais qu'on guette et qu'on attend aussi comme du 10 au 15 juin 1814, presque à l'heure de Waterloo donc, où Mansord note successivement : il y a «un drapeau blanc sur la fontaine aux Herbes"; "L'aigle impériale n'est point encore à la porte de la préfecture", mais il y est le lendemain; "Dans la nuit, on a enlevé les armes du roi sarde de dessus la porte d'entrée du château»; peu avant il notait : "On a fait quelques conjectures de ce que le drapeau de Savoie avait été retiré du balcon de la mairie. C'est tout bonnement à cause de la pluie». Il en va de même des chants : La Marseillaise, le Ça ira ou Veillons au salut de l'Empire, assurent Mansord que les Cent-Jours ont bel et bien débuté à Chambéry. Tous les mouvements sont épiés, et tout spécialement les incessantes allées, venues et passages : depuis ou vers Turin ou Paris. La période se distingue par une forte mobilité des hommes, des nouvelles, des symboles officiels et partisans.

22. Sur les mécaniques de la rumeur, $c f$. François Ploux, De bouche à oreille. Naissance et propagation de la rumeur dans la France du XIX' siècle, Paris, Aubier, 2003. 
Durant deux ans, Chambéry ne cesse d'être animée par le passage des troupes qui retient toute l'attention et qui est une autre source d'information, donc d'interrogation : celles qui refluent, celles qui stationnent à Chambéry; celles qui n'y font que passer, celles qui s'installent cérémonieusement; les soldats en débandade ou déserteurs qui, depuis la France ou l'Italie du nord, passent par petits groupes. L'auteur décrit avec précision les uniformes des régiments autrichiens, français, sardes et suggère par là l'instabilité de l'encadrement du quotidien car la plupart de ces troupes stationnent quelques jours puis partent et sont remplacées par d'autres contingents, ennemis ou non. Bien entendu, elles sont source d'inquiétude car il faut les entretenir, mais cette militarisation du paysage quotidien semble soulever plus de curiosité que d'angoisse : le nombre d'hommes, leur départ et arrivée, leur équipage - le nombre de pièces d'artillerie, de chevaux, de sapeurs semble pour Mansord un signe en mesure d'informer sur les opérations en cours - sont d'abord des moyens d'interpréter les événements.

Sans doute l'avocat n'est-il pas dupe de la valeur des nouvelles qu'il rapporte et, à chaque page, toutes sont accompagnées de la mention «On dit que...", sans que la source soit généralement identifiée. Il est curieux de noter l'attention de Mansord - on voit bien qu'il ne s'agit pas que de la sienne propre, mais bien d'une attitude collective, au moins dans la classe "éclairée" - à tous ces signes et surtout à toutes les rumeurs qu'il rapporte avec une troublante obstination pendant près de deux ans, sans pour autant que ces «renseignements» ne lui servent vraiment d'instruments d'analyse. Le journal ne laisse place à aucune notation personnelle. Il consigne les bruits comme il consigne le prix du froment et la météorologie : des faits chaque jour un peu variables, recensés avec la même sérénité. Son auteur résume parfaitement la situation en une formule lapidaire : «On a bien tant dit des choses aujourd'hui qu'à la fin on n'en sait rien sauf ce que l'on voit».

Ce journal témoigne du besoin de consigner un temps «extraordinaire» et mieux qu'une analyse, il révèle le desserrement et l'ouverture du champ des possibles que provoquent la guerre et les changements de régimes. Cette attention à la valse des couleurs, des troupes et des rumeurs montre surtout que la Savoie éprouve une certaine "plurinationalité», sentiment renforcé par le décalage entre les événements internationaux et leur traduction locale ${ }^{23}$, ainsi que la succession de fêtes contradictoires : les Autrichiens ont fêté publiquement leur prince; mais on fête bientôt la Saint-Louis; on renoue sous les Cent-Jours avec les chants de la Grande Nation; on bénit ensuite les drapeaux blancs en grande pompe à la cathédrale et on organise un banquet

23. La Savoie vit toute la période un peu «à coutre-coup", en décalage, au rythme d'une zone de contact. Cette distorsion entre l'état de la situation à Paris, centre des nouvelles officielles, et la réalité des pouvoirs locaux contribue à expliquer l'attitude de Mansord qui cherche la traductionconfirmation des changements dans «son» espace. 
pour le roi de France; on fête enfin le retour du roi légitime et des troupes sardes, etc.

Il est difficile de se faire une idée précise de la participation des populations à ces fêtes convenues et on ne peut pas trop à ce sujet se fier aux transports de joie consignés par des témoins rarement désintéressés. Toutefois, les Savoyards, sans doute pas tous avec la même intensité que ceux de Chambéry, subissaient concrètement les rituels politiques, martiaux et dynastiques et pouvaient d'autant moins ignorer que leur destin était incertain qu'ils furent sollicités par les partis opposés. Les divergences d'opinions s'exprimaient publiquement dans un département du Mont-Blanc où ni les Autrichiens ni le préfet napoléonien n’arrivaient à contrôler l'élite : «On voit [les officiers du roi de Sardaigne], revêtus de leurs uniformes, exprimer hautement leurs espérances dans les caffés [sic], sur les places et même assez indiscrètement pour être entendus des militaires rappelés et des gens de la campagne ${ }^{24}$. Cette rivalité au sein des élites, encouragée par le contexte d'incertitude, se prolonge tout au long de la période et, selon les mots du préfet, «cette espèce de discussion intérieure, inaperçue partout ailleurs, n'en est pas moins pénible pour l'administrateur qui placé entre la susceptibilité du premier parti et l'exigence du second ne fait plus une seule démarche qui soit sans conséquences ${ }^{25}$. Les conséquences de cette rivalité doivent toutefois être d'autant moins exagérées qu'elle devait finalement se résorber devant la «question nationale».

\section{LA SOLUTION DE LA QUESTION NATIONALE SERA RÉACTIONNAIRE}

On ne saurait prétendre que les populations furent massivement conviées à choisir leur destin entre Paris et Turin. L'aristocratie pro-sarde a mis d'abord en mouvement, dès le début de 1814 , ses réseaux internationaux vers les alliés, vers le roi de Sardaigne et vers la France. Le parfum de la Restauration est très sensible dans l'adresse des «dames du duché de Savoie à S.A.R. Madame la duchesse d'Angoulême", où ces dames réclamaient l'appui de la duchesse pour qu'elles retrouvent le père que 1792 leur avait enlevé ${ }^{26}$. Néanmoins, ce type de démarches et les conciliabules confidentiels ne l'étaient volontairement pas assez pour ne pas nourrir les rumeurs : celles-ci sont un vrai levier politique et on comprend sous cet angle l'importance que leur accorde Mansord. Le partage de la Savoie qui fait suite au premier traité de Paris a encouragé, parce qu'il était considéré comme absurde par tous les partis savoyards, à rendre publiques des démarches qui se voulaient d'abord plus

24. Arch. nat., FibI Mont-Blanc 4, lettre du préfet Finot, 5 décembre 1814.

25. Arch. nat., FlcIII Mont-Blanc 3, le préfet Finot à Montesquiou, janvier 1815.

26. Reproduite dans : Tredicini de Saint-Séverin, Souvenirs de 1814. Le général comte Janus de Gerbaix de Sonnaz d'Habères et les volontaires savoyards, Genève, Trembley, 1890, p. 85. Adresse accompagnée de 233 signatures. 
discrètes. Cette ligne de partage ne mettait pas seulement à mal les intérêts des grands propriétaires : il existait donc un intérêt commun qui légitimait l'argument nationalitaire et l'invocation larvée du vœu des populations.

Dès le début des opérations militaires, les parties en présence ont fait vibrer la corde patriotique lors des tentatives de mobilisation : pour résister à l'envahisseur autrichien ${ }^{27}$; pour au contraire l'épauler ${ }^{28}$; à nouveau lors des Cent-Jours pour mobiliser autour de l'Empereur ${ }^{29}$. Les forces se disputaient clairement l'identité d'un peuple savoyard, qui d'ailleurs faisait surtout la sourde oreille. Conjoncturel, cet appel à la mobilisation devait se prolonger durant toute la période sous l'invocation moins martiale d'une "nation savoyarde» qui devint pour toutes les opinions la légitimité de leurs prétentions pour l'avenir de la Savoie. On se limitera ici à citer une des premières adresses, très officielle ${ }^{30}$. Datée d'avril 1814, avant le partage donc, elle est issue de la Commission centrale mise en place par les Autrichiens et, rédigée alors que la Savoie toute entière paraît vouée à revenir au roi de Sardaigne, elle était destinée à ce dernier via le général Bubna. Ses membres exprimaient sur un mode pour le moins emphatique la joie de retrouver leur souverain avec toutefois des réserves en des termes qu'il convient de souligner : la Savoie a toujours formé "un corps de nation»; les sujets de Napoléon ont été déliés de leur serment, ce qui restitue aux Savoyards «la liberté politique qu'ils avaient [avant la réunion à la France] et [les rend] libres d'exprimer leur vœu soit pour la forme de gouvernement, soit pour la dynastie qu'ils croient la plus propre à leur bonheur ». À cet égard, l'oubli du passé ne peut se concevoir que sur la base des mêmes institutions que celles qui régissent la Restauration en France. On ne saurait être complètement surpris de ces réserves de la part d'une commission qui reste composée de notabilités impériales, mais il y figure aussi des membres de la haute noblesse, comme Salteur de la Serraz ${ }^{31}$, peu suspect d'hostilité au principe de restauration. En réalité, l'argument du vœu national visait à mettre en valeur la singularité de la Savoie et tout spécialement à désamorcer la bombe que constituait la question des biens nationaux, plus qu'ailleurs peut-être compliquée $^{32}$ : «et que toutes les propriétés, sans exception, soient maintenues, et que tout le passé soit oublié», est-il bien précisé. En définitive, la mise en exergue de la nationalité savoyarde a donc pour fonction de pacifier et de

27. Par exemple, circulaire préfectorale aux maires reproduite dans la Gazette du Mont-Blanc, 10 janvier 1814.

28. L’appel de Sonnaz du 17 janvier 1814, déjà évoqué.

29. L'appel de Constant Durhône, op. cit.

30. Arch. dép. Savoie, L 1429, arrêtés de la Commission du Mont-Blanc, 10 avril 1814.

31. César Salteur de la Serraz (1751-1833), issu d'une grande famille noble savoyarde, emprisonné sous la Révolution comme suspect. Président du Conseil général du Mont-Blanc de 1802 à 1814 .

32. La question des biens nationaux était particulièrement sensible en Savoie parce que les émigrés saisis avaient rapidement contesté leur statut d'émigrés, en soulignant qu'en 1792 ils avaient obéit à leur prince légitime. La plupart des membres de la noblesse sont de retour à partir de 1796 (armistice de Cherasco) et reprennent leur place dans le duché de Savoie. Il n'existe pas d'analyse précise de l'émigration en Savoie. 
désamorcer les tensions sociales : en l'espèce, la question du devenir des biens nationaux de seconde origine, dont la vente avait profondément fragilisé certaines fortunes nobiliaires dans la Savoie annexée à la France (à la différence de la situation dans le Piémont) et impliquait un traitement particulier pour le duché réintégré dans un royaume qui postulait d'emblée une restauration intégrale ${ }^{33}$. L'invocation de la nationalité savoyarde se justifiait donc pour toutes les forces en présence.

Dès avril 1814, la noblesse pro-sarde avait pris l'initiative de s'appuyer sur les populations quand Joseph de Sonnaz avait porté à Victor-Emmanuel des adresses des communes du nord de la Savoie ${ }^{34}$. Ces adresses, dispersées dans les archives ${ }^{35}$, se renouvellent tout au long de la période, incroyablement nombreuses : aux autorités autrichiennes, voire anglaises et russes; au roi de Sardaigne, à Louis XVIII, à Napoléon lors des Cent-Jours, au Congrès de Vienne. Exercice convenu lors des changements de souveraineté, elles cherchaient aussi à modifier l'ordre politique, prenant parfois la forme de mémoires ${ }^{36}$, et lançaient sur les routes des délégués de toutes sortes - peu taiseux, ce sont ceux qu'épie Mansord. Par exemple, en juin 1814, des députés de la Savoie du Nord portent les adresses des trois provinces, au total 577 signatures, à Zurich pour demander leur rattachement à Genève ${ }^{37}$. Sans doute ne faut-il pas trop s'illusionner sur le fondement démocratique de ces démarches. Toutefois, elles portaient expressément avec elles l'idée de vœux des populations, toujours convoquées comme forme de légitimité. D'ailleurs les idées qu'elles développaient étaient livrées à la publicité. On peut à ce titre mettre en parallèle deux écrits parfaitement contraires, et soulignons que Charles Mansord les connaissait : l'opuscule de l'ancien député Anthelme Marin $^{38}$ et celui, anonyme, intitulé sobrement Notice sur la Savoie $e^{39}$. Le premier plaide pour le droit des Savoyards à jouir de leurs efforts des dernières années, à savoir de vivre sous le régime sagement constitutionnel de la

33. Le gouvernement sarde devait semble-t-il tenir compte de ces difficultés puisqu'il opta en 1818 pour une indemnisation assez massive des familles dont les biens avaient été vendus, entérinant par là l'irrévocabilité du transfert de ces biens (tableau de ces indemnités: Arch. dép. Savoie, 1Q 181).

34. Tredicini de Saint-Séverin, Souvenirs de 1814..., op. cit., p. 64.

35. Ces adresses, souvent mentionnées, paraissent en effet avoir été très nombreuses, mais sont dispersées dans les fonds d'archives publiques (où elles ne sont d'ailleurs pas toutes conservées) ainsi que dans des fonds familiaux. Tredicini, op. cit., en cite intégralement un certain nombre.

36. André Palluel-Guillard dans Une fusion manquée... (op. cit., tome II, p. 1407) évoque quatre mémoires portés à Paris et accompagnant la démarche pétitionnaire. Il y en eut d'autres sans doute et on comprend bien l'intérêt que Mansord porte aux mouvements de ces notables.

37. Sur cette question suisse, $c f$. Luc Monnier, "La Savoie du Nord et Genève en 1814 ", Bulletin de la Société d'histoire et d'archéologie de Genève, 1956, p. 23-45; André Palluel-Guillard, Une fusion manquée..., op. cit., tome II, p. 1289 et suiv.; Paul Guichonnet, La Savoie du Nord et la Suisse, Chambéry, SSHA, 2001. Voir aussi : Réflexion d'un Suisse sur l'état actuel de la Savoye, telle qu'elle se trouve placée par le traité de paix du 30 mai 1814, Chambery, Gorrin, s.d., 7 p.

38. Anthelme Marin, Les Alpes sont les limites naturelles et nécessaires du territoire français, Chambéry, Gorrin, 1814, 20 p. Anthelme Marin (1760-1825), avocat, jacobin distingué, fut député à la Convention et au Conseil des Cinq-Cents, avant d'entamer une courte carrière de professeur.

39. Notice sur la Savoie précédée d'une carte topographique, Paris, Michaud, 1815, 42 p. Mémoire attribué à Jean-Baptiste d'Oncieu. 
France. Il convoque pour se justifier, la logique des frontières naturelles - les Alpes évidemment, avec pour corollaire la paix européenne -, l'histoire - les ducs de Savoie n'ont gardé le duché que quand ils étaient alliés à la France - et la "culture» du peuple - sa langue, ses mours : "plusieurs siècles de réunion sous la même domination n'ont pas pu vaincre l'éloignement qui existe entre les Savoisiens et les Piémontais». En fin de compte les années révolutionnaires et impériales ont permis de définir les lois d'appartenance nationale que la nature impose : "On voudrait effacer de nos âmes ce sentiment de gloire et de grandeur qu'il nous est permis de partager avec tous les Français, puisque nous avons partagé avec eux le poids de leurs peines et de leurs privations». La Notice sur la Savoie propose le même argumentaire, mais en négatif. Une frontière naturelle nette, consacrée par le temps : le Rhône; des intérêts géostratégiques : une population montagnarde appuyée sur des bastions naturels dont les Romains ont pu éprouver la résistance; une histoire : celle d'un État de huit siècles d'existence et d'une «illustre maison à laquelle [les habitants de la Savoie] ont donné leur nom»; enfin, l'auteur évoque la liesse populaire qui accueillit les premiers contingents de l'armée sarde dans le territoire restitué, la couleur bleue de retour - on voit encore l'importance des insignes arborés dans l'espace public. En clair, «nous entendons tous les jours l'opinion générale», une opinion qui est bien celle du "plus grand nombre».

Ces deux argumentaires allient de manière inextricable les intérêts pour l'équilibre de l'Europe et les intérêts locaux. Or, ces derniers sont clairement articulés avec un argumentaire de type nationalitaire, proche de ce que synthétisera plus tard Renan : une nation c'est un territoire, la volonté de vivre ensemble, un passé commun. La conception de ce dernier est finalement le point qui divisait. Ceux qui défendaient le rattachement à la Restauration sarde utilisaient sans rechigner des logiques nées de la Révolution en s'appuyant chaque fois sur la convocation d'un assez vague consentement populaire : outre les manifestations de liesse déjà évoquées - que la Restauration aura soin de médiatiser en $1816^{40}$-, il est spécifié ainsi dans la Notice que pas $1 / 20^{\text {e }}$ de la population savoyarde ne penche pour la frontière au Mont-Cenis.

Les autorités autrichiennes en 1814 comme en 1815 se sont efforcées de mettre le holà à cette publicité ainsi que, si l'on en croit Mansord, à cette idée de vœux : «on dit que M. de Bubna avait répondu [à une délégation qui l'avait rencontré] que les habitants d'un pays conquis n'avaient point à s'assembler, ni de vœu à exprimer ou à faire exprimer par députation sans l'autorisation du conquérant». Cette difficulté à se faire entendre, envenimée par le partage de la Savoie, a amené les partisans du rattachement au royaume de Sardaigne à franchir un pas à l'été 1815 en initiant ce qu'il a été

40. Relation des fêtes qui ont eu lieu à Chambéry les 16 et 17 décembre 1815 et les circonstances qui les ont précédées, slsn, 1815, 53 p. 
convenu d'appeler par la suite le "plébiscite occulte ${ }^{41}$ : une consultation qui fut portée à Paris, puis transmise à Turin pour preuve de fidélité. Au total, 27259 chefs de famille auraient fait connaître leur opinion et 820 seulement se seraient opposés au retour de la Savoie au roi de Sardaigne. Toutes les paroisses du département, soit 244, sont concernées. C'est à bon droit que Max Bruchet, archiviste départemental de la Haute-Savoie - le seul qui ait travaillé sur ces sources conservées à Turin - critique la signification de ce "plébiscite», dont le récolement des signatures paraît assez opaque. Bruchet accuse le clergé d'avoir été l'agent local de cette "consultation populaire». Toutefois, en cherchant à prouver comment ces résultats prouvent finalement en creux l'existence d'un parti français conséquent, l'auteur manque son but : il en fait dire plus qu'il ne signifiait à ce plébiscite. Il ne s'agissait nullement d'une consultation démocratique; ses promoteurs se revendiquaient d'une logique de type censitaire, considérant que sur les 180000 habitants du département, seuls 31676 chefs de famille étaient à même de former le corps «électoral».

En fait, ce qui importe dans l'optique qui est la nôtre, c'est que l'aristocratie et le clergé ont opté, dans le cadre de l'Europe du Congrès de Vienne naissante, pour le principe du droit des peuples à disposer d'eux-mêmes qu'ils ont considéré être un argument devant le blocage que constituait le partage d'une "nation savoyarde qui pour être petite, n'en est pas moins estimable", suivant les termes de la circulaire à l'appui de la campagne de signatures ${ }^{42}$. Si la démarche ne fait pas l'unanimité dans la noblesse - Paul de Sales critique ce «moyen qui ne signifie rien à présent ${ }^{43}-$, elle a en revanche été unanimement appuyée par le clergé qui avait d'ailleurs effectué un premier pétitionnement, de moindre envergure, dès le printemps $1814^{44}$. Les grandes familles, comme les Costa de Beauregard, se servent de ces registres dans leurs démarches à Paris. Ce sont donc au moins une partie des partisans de la Restauration la plus stricte, et ses soutiens durables, qui ont usé à la fois de l'argumentaire autour des droits de la nationalité et des pratiques consultatives.

Cette bizarrerie est moins surprenante si on se rappelle que la Savoie, premier territoire annexé à la France révolutionnaire en 1792, l'avait été suite à un vœu émis par sa population, ou du moins par ses représentants regroupés dans l'assemblée des Allobroges chargée d'administrer révolutionnairement la Savoie occupée par les armées françaises. C'était alors le choix de 660 députés, élus un peu à la hâte. Il est assez évident que les promoteurs du "plébiscite occulte» cherchaient à annuler le vote de 1792, dont ils conser-

41. Max Bruchet, "Le plébiscite occulte du département du Mont-Blanc en 1815 et la Restauration en Savoie", Bulletin historique et philologique, 1902, p. 329-371. Des compléments dans : André Palluel-Guillard, Une fusion manquée..., op. cit., tome II, p. 1398 et suiv.

42. Max Bruchet, «Le plébiscite occulte»..., art cité., p. 355.

43. Cité dans André Palluel-Guillard, Une fusion manquée, op. cit., tome II, p. 1401.

44. Idem, tome II, p. 1279 et suiv. 
vaient une mémoire d'autant plus claire que les partisans de la France ne manquaient pas de le leur rappeler, au moins à demi-mot - la "réunion à la France est naturelle et nécessaire, elle fut le résultat de considérations longtemps méditées " ${ }^{45}$. La fonction de cette consultation s'éclaire encore mieux si on songe que les Savoyards ont voté récemment : ils ont élu leurs députés en mai 1815 (avec un taux de participation supérieur à 60\%) et ont été de nouveau sollicités pour l'approbation de l'Acte additionnel. Non seulement donc, les vingt ans de régime français ne sont pas passés sans laisser de traces, mais la «volonté populaire» fait désormais sens pour ces hommes qui préparent ardemment une Restauration en rien comparable avec ce qu'elle sera en France. Simple tactique? Ce n'est pas sûr. On ne jugera pas ici de l'efficacité de ce type de démarche auprès des puissances, mais on notera que pour les grands notables de la Savoie comme pour le clergé - dont il faut rappeler qu'il est exclusivement "endogène" -, cette procédure ne fait que renforcer leur conception de l'ordre social : ils voient dans cet appui populaire la confirmation des hiérarchies naturelles et de leur position retrouvée. Du reste, le principe du vote n'implique pas mécaniquement la démocratie ${ }^{46}$.

Les années qui suivirent les consolidèrent pour partie dans le sentiment d'être, certes les fidèles sujets de roi, mais aussi les représentants d'une petite nation. L'expérience de 1814-1815 devait d'ailleurs se reproduire : ce sont bien les grands notables conservateurs et le clergé qui, en 1860, à grands coups d'adresses et par l'encadrement du plébiscite napoléonien, contribuent à orner des parures de la souveraineté l'annexion de la Savoie à la France. Au nom des intérêts "savoisiens", au nom de l'ordre, au nom aussi d'un refus de démembrement de l'ancien duché entre cette fois la France et la Suisse.

L'argumentaire nationalitaire, bien réel et patent, put se développer pour deux raisons majeures et conjoncturelles : d'une part, le desserrement administratif, l'atmosphère de flottement, bien sensible dans le journal de Mansord, qui permit sur une durée assez longue d'interroger le devenir du duché et de rendre pensable le possible; d'autre part, le partage de la Savoie qui ne trouva aucun partisan. Sur ce terreau pouvait prendre une propagande contradictoire et les idées de la Révolution sur la souveraineté continuer de faire leur chemin par d'autres voies, certes plus étroites. Le retour intégral de la Savoie à la France étant proprement impensable au regard de la stratégie des alliés, un des deux partis eut rapidement l'avantage sur l'autre. Les modes de règlements, au niveau local, de la question de l'appartenance nationale que soulevait la situation créée en 1814 , posaient finalement les conditions

45. Appel de Constant Durhône, op. cit.

46. Comme cela a été récemment et précisément rappelé : Oliver Christin, Vox Populi : Une histoire du vote avant le suffrage universel, Paris, PUF, 2014. 
d'un pacte social nouveau. De ce point de vue, il faut noter que la division de la Savoie rapprocha dans l'organisation du "plébiscite occulte» comme dans celle des différentes députations, l'élite issue du régime français et l'aristocratie farouchement pro-sarde : d'Oncieu, le maire de Chambéry, appartenait à l'une et à l'autre et il devint l'un des organisateurs du "plébiscite occulte». Le réalisme devait assoupir les divergences politiques. En réalité, l'aristocratie progressivement rétablie - au moins pour partie - dans ses propriétés depuis 1796 ainsi que les acheteurs de biens nationaux ne pouvaient souscrire au partage de la Savoie pour des questions d'intérêts bien compris. Curieusement, ce partage créa les conditions de la fusion notabiliaire que Napoléon avait tenté, et qui avait débuté à partir de $1810^{47}$. Ce n'est pas le lieu d'évoquer ici le destin de ces grands notables du duché, mais la plupart d'entre eux s'allièrent par la suite - matrimonialement parlant ou dans des entreprises de modernisation du territoire - pour la maîtrise de la petite société savoyarde, et sans véritables heurts jusqu'en $1848^{48}$. L'argument nationalitaire aurait donc participé en Savoie à l'issue pacifique du transfert de nationalité et des logiques profondes se dessinent sous les discontinuités des régimes politiques : on se revendiquait de l'opinion.

Sylvain Milbach est maître de conférences $H D R$ à l'Université de Savoie

47. Jean Nicolas, «Le ralliement des notables à l'Empire dans le département du Mont-Blanc», Revue d'histoire moderne et contemporaine, 1972, p. 92-127.

48. Citons simplement le cas de Marin, conventionnel, à la tête d'une belle propriété au sortir de l'Empire et qui prend place ensuite parmi les agronomes distingués et dans le réseau de la haute notabilité savoyarde. Ses fils et petit-fils poursuivent leur œuvre, ornés d'un titre de baron décerné par Charles-Félix : réputée pour leur soutien aux œuvres catholiques, cette famille se classe après 1848 dans les rangs de la notabilité conservatrice aux cotés des grands noms de l'aristocratie ancienne. 